\title{
Gapped dilatons in scale invariant superfluids
}

\author{
Riccardo Argurio, ${ }^{1, *}$ Carlos Hoyos $\odot,,^{2, \dagger}$ Daniele Musso $\odot,{ }^{3, *}$ and Daniel Naegels $\circledast^{1, \S}$ \\ ${ }^{1}$ Physique Théorique et Mathématique and International Solvay Institutes, \\ Université Libre de Bruxelles, C.P. 231, B-1050 Brussels, Belgium \\ ${ }^{2}$ Department of Physics and Instituto de Ciencias y Tecnologías Espaciales de Asturias (ICTEA), \\ Universidad de Oviedo, c/ Federico García Lorca 18, E-33007 Oviedo, Spain \\ ${ }^{3}$ Departamento de Física de Partículas and Instituto Galego de Física de Altas Enerxías (IGFAE), \\ Universidade de Santiago de Compostela, E-15782 Santiago de Compostela, \\ Spain Inovalabs Digital S.L. (TECHEYE), E-36202 Vigo, Spain
}

(Received 9 July 2020; accepted 25 September 2020; published 19 October 2020)

\begin{abstract}
We study a paradigmatic model in field theory where a global $U(1)$ and scale symmetries are jointly and spontaneously broken. At zero density the model has a noncompact flat direction, which at finite density needs to be slightly lifted. The resulting low-energy spectrum is composed by a standard gapless $U(1)$ Nambu-Goldstone mode and a light dilaton whose gap is determined by the chemical potential and corrected by the couplings. Even though $U(1)$ and scale symmetries commute, there is a mixing between the $U(1)$ Nambu-Goldstone and the dilaton that is crucial to recover the expected dynamics of a conformal fluid and leads to a phonon propagating at the speed of sound. The results rely solely on an accurate study of the Ward-Takahashi identities and are checked against standard fluctuation computations. We extend our results to a boosted superfluid, and comment the relevance of our findings to condensed matter applications.
\end{abstract}

DOI: 10.1103/PhysRevD.102.076011

\section{INTRODUCTION}

Scale invariance plays a special role in many-body and high-energy physics. It underlies the emergence of universality in many instances, such as critical phenomena, Landau-Fermi liquids or cold atoms at unitarity, to name a few. Scale transformations are a symmetry either at very low or very high energies compared to the intrinsic scales. In most cases they represent only an approximate symmetry valid in a restricted regime, requiring typically a certain degree of fine-tuning in the interactions, the thermodynamic variables, the external parameters, or the support of additional symmetries. When a scale invariant system is considered at non-zero particle number or at finite charge density, scale symmetry is spontaneously broken; such breaking is directly relevant to characterize the dynamics of the system mentioned above but it can also be useful to

\footnotetext{
rargurio@ulb.ac.be

thoyoscarlos@uniovi.es

*daniele.musso@usc.es

§aniel.naegels@ulb.ac.be
}

Published by the American Physical Society under the terms of the Creative Commons Attribution 4.0 International license. Further distribution of this work must maintain attribution to the author(s) and the published article's title, journal citation, and DOI. Funded by SCOAP ${ }^{3}$. extract properties of large charge operators of a CFT via the state-operator correspondence [1-4].

Whenever an internal symmetry is spontaneously broken in a relativistic system, one expects to encounter gapless excitations in the form of Nambu-Goldstone (NG) modes [5-7], one for each broken symmetry. If instead the breaking involves spacetime symmetries, the counting of modes becomes more complicated [8-11], yet the presence of a Nambu-Goldstone mode associated to scale invariance, commonly known as dilaton, is still a possibility. Similarly, the counting of NG modes deviates from the standard Goldstone theorem expectation when the system is not Lorentz invariant [10,12-15]. ${ }^{1}$

Even in relativistic systems, the presence of a nonzero charge density breaks the boosts spontaneously, thus the NG modes may show some features similar to those emerging in nonrelativistic systems. In the case of several internal symmetries, there can be additional gapped modes besides the gapless NG modes [17-22]. More specifically, in the presence of a chemical potential $\mu$ for a conserved charge $Q$, gapped modes emerge when the effective Hamiltonian $^{2} \tilde{H}=H-\mu Q$ does not commute with the broken generators. The gap is fixed by group theory considerations and is proportional to the chemical potential.

\footnotetext{
${ }^{1}$ For a recent review on NG counting rules we refer to [16].

${ }^{2} \mathrm{We}$ are proceeding in analogy to $[19,20,22,23]$.
} 
In general, there can also be additional modes whose gap, although proportional to $\mu$, is not protected by symmetry $[15,21]$. Such analysis was generalized in [20] to cases where $\mu Q$ in the effective Hamiltonian is replaced by some other deformation involving a symmetry generator, for instance the magnetic field times the spin in a ferromagnet $\tilde{H}=H-g B_{z} S_{z}$.

The breaking of scale invariance presents some similarities with the story above due to the fact that the generator of dilatations $D$ does not commute with the Hamiltonian $[D, H]=i H .^{3}$ Accordingly, the commutator with the effective Hamiltonian is

$$
[\tilde{H}, D]=-i H
$$

For simplicity let us assume that $Q$ is the generator of an Abelian $U(1)$ symmetry. If this symmetry is spontaneously broken, the ground state is not an eigenstate of $Q$. However, it must be by definition an eigenstate of $\tilde{H}$, so time translations generated by $H$ are spontaneously broken too. In fact time translations and the $U(1)$ symmetry are broken to a diagonal subgroup and there is just a single NG mode associated to both generators.

Equation (1.1) implies that there is a mixing between the $U(1) \mathrm{NG}$ and the dilaton, then - even though $Q$ commutes with $\tilde{H}$-the state produced by the corresponding charge density $J^{0}$ applied to the vacuum at some initial time is not an eigenstate of time evolution. Borrowing an analogy from high-energy physics, the "flavor" eigenstates defined by the symmetry generators are not aligned with the "mass" eigenstates. If the dilaton were not dynamical, or if it were integrated out, the mixing implied by (1.1) would be manifested in the form of an inverse Higgs constraint.

Although interesting, one might wonder whether it is sensible to discuss the physics of a dilaton in the first place, since the energy density is in general nonzero at nonzero charge density. In that case, a scale transformation would change the vacuum energy density (as determined by the temporal component of the energy-momentum tensor $T^{\mu \nu}$ ) by an amount proportional to itself

$$
\delta\left\langle T^{00}\right\rangle \sim\left\langle-i\left[D, T^{00}\right]\right\rangle=(d+1)\left\langle T^{00}\right\rangle,
$$

where $d+1$ is the number of spacetime dimensions. Both here and henceforth, we assume a relativistic theory, thus there cannot be a NG mode associated to the spontaneous breaking of scale invariance unless $\left\langle T^{00}\right\rangle=0$. ${ }^{4}$ This is quite restrictive. Since a gapless mode requires a degeneracy of

\footnotetext{
${ }^{3}$ Nevertheless, it is a conserved charge because $\partial_{t} D=H$, so its total time derivative in the Heisenberg picture vanishes.

${ }^{4}$ Note also that the combination of Lorentz invariance (which fixes the expectation value of the energy-momentum tensor to $\left.\left\langle T^{\mu \nu}\right\rangle=\Lambda \eta^{\mu \nu}\right)$ and the Ward-Takahashi identity for scale invariance, $\left\langle T^{\mu}{ }_{\mu}\right\rangle=0$, fixes $\left\langle T^{00}\right\rangle=0$.
}

ground states, the theory needs to have a moduli space of vacua in addition to scale invariance: these are flat directions in the potential, supposing we refer to a field theory with a Lagrangian. ${ }^{5}$

Maybe contrary to expectations, the situation at finite density is similar despite the fact that the energy density is nonvanishing. If the ground state is homogeneous and isotropic, the expectation value of the components of the energy-momentum tensor correspond to constant energy density and pressure

$$
\left\langle T^{00}\right\rangle=\varepsilon, \quad\left\langle T^{i j}\right\rangle=p \delta^{i j} .
$$

Scale invariance implies that the expectation value of the trace of the energy-momentum tensor will vanish $\left\langle T^{\mu}{ }_{\mu}\right\rangle=0$, which fixes the equation of state $\varepsilon=d p$, where $d$ is the number of spatial dimensions. In addition, we have the usual relation between thermodynamic potentials at zero temperature, $\varepsilon+p=\mu \rho$, where $\rho=\left\langle J^{0}\right\rangle$ is the $U(1)$ charge density. Combining the two, the energy density of the scale invariant theory is $\varepsilon=d /(d+1) \mu \rho$. At finite density the relevant quantity is not the energy density, but the free energy (density) given by the effective Hamiltonian $T^{00}-\mu J^{0}$. A scale transformation changes the expectation of the effective energy density as follows:

$$
\begin{aligned}
\delta\left\langle T^{00}-\mu J^{0}\right\rangle & \sim\left\langle-i\left[D, T^{00}\right]\right\rangle-\mu\left\langle-i\left[D, J^{0}\right]\right\rangle \\
& =(d+1) \varepsilon-d \mu \rho=0 .
\end{aligned}
$$

Then, under quite general assumptions, scale transformations do not shift the free energy of a finite density state in a scale invariant theory and it is legitimate to discuss the physics of a dilaton mode, at least at zero temperature.

The observation above does not imply directly the existence of a gapless (or gapped) mode. In the absence of a general argument that would allow us to fix the properties of a dilaton mode, we study a concrete model of spontaneous breaking of scale invariance at nonzero density. We restrict the analysis to a relativistic theory in $3+1$ dimensions, and keep the analysis classical. Such simple model is informative because it can be interpreted as an effective action $\grave{a}$ la Ginzburg-Landau for the order parameter.

The principal highlights of the present study are two. On one side is the characterization of the dilaton dispersion relation and particularly its gap. This concerns mainly the effects of the chemical potential and its role in defining the effective low-energy spectrum. On the other side, we propose and check a method based uniquely on the study of Ward-Takahashi identities, that in our setup just correspond to classical conservation equations.

\footnotetext{
${ }^{5} \mathrm{~A}$ related discussion about fine-tuning the cosmological constant to zero in order to have a flat dilatonic direction is contained in [24-27].
} 
The paper is structured as follows. Section II introduces the model at zero density, where we emphasize the need for flat directions in the potential. This condition is relaxed in Sec. III, where we study the model at nonzero density. In Sec. IV the analysis is extended to allow for nonzero superfluid velocity. Each section has a subsection dedicated to the analysis of the Ward-Takahashi identities, together with a check of the latter method against standard Lagrangian computations for the fluctuations. We conclude the paper in Sec. V with further comments on the results, their interpretation, their applications and possible extensions.

\section{THE MODEL}

Consider the standard Goldstone model for a global $U(1)$ symmetry in four spacetime dimensions

$$
S=\int d^{4} x\left[\partial_{\mu} \psi \partial^{\mu} \psi^{*}-\lambda\left(|\psi|^{2}-v^{2}\right)^{2}\right],
$$

where $\psi$ is a scalar complex field charged under the $U(1)$ symmetry, which acts as $\psi \rightarrow e^{i \alpha} \psi$, while $\lambda$ and $v$ represent-respectively - a dimensionless and a dimensionful coupling. Given the presence of a dimensionful coupling, the model (2.1) does not enjoy scale invariance. We can nonetheless make it scale invariant if we replace $v$ with a dynamical real scalar field $\xi$ acting as a compensator:

$$
S=\int d^{4} x\left[\partial_{\mu} \psi \partial^{\mu} \psi^{*}+\frac{1}{2} \partial_{\mu} \xi \partial^{\mu} \xi-\lambda\left(|\psi|^{2}-\xi^{2}\right)^{2}\right] .
$$

The equations of motion are given by

$\partial^{2} \psi+2 \lambda\left(|\psi|^{2}-\xi^{2}\right) \psi=0, \quad \partial^{2} \xi-4 \lambda\left(|\psi|^{2}-\xi^{2}\right) \xi=0$,

and the generic stationary solution is

$$
\xi=v, \quad|\psi|^{2}=v^{2}
$$

The space of solutions (2.4) has two moduli, $\xi$ itself and the phase of $\psi$. Consider the fluctuations around (2.4), parametrized as follows

$$
\begin{aligned}
& \psi=e^{i \frac{\vartheta}{\sqrt{2 v}}}\left(v e^{\frac{\tau}{\sqrt{3} v}}+\frac{\rho}{\sqrt{6}}\right) \simeq v+\frac{\tau}{\sqrt{3}}+\frac{\rho}{\sqrt{6}}+i \frac{\vartheta}{\sqrt{2}}, \\
& \xi=v e^{\frac{\tau}{\sqrt{3 v}}}-2 \frac{\rho}{\sqrt{6}} \simeq v+\frac{\tau}{\sqrt{3}}-2 \frac{\rho}{\sqrt{6}},
\end{aligned}
$$

where $\tau, \rho$ and $\theta$ are real. The quadratic action for the fluctuations is given by
$S_{\text {quad }}=\int d^{4} x\left[\frac{1}{2} \partial_{\mu} \tau \partial^{\mu} \tau+\frac{1}{2} \partial_{\mu} \rho \partial^{\mu} \rho+\frac{1}{2} \partial_{\mu} \vartheta \partial^{\mu} \vartheta-6 \lambda v^{2} \rho^{2}\right]$.

We thus see that $\rho$ gets a mass $12 \lambda v^{2}$ while $\tau$ and $\vartheta$ are massless. We identify the latter two with the Goldstone bosons for broken scale invariance, the dilaton and, for broken $U(1)$ symmetry, the $U(1)$ NG. The dispersion relations are trivially relativistic, since Lorentz symmetry is preserved.

In order to study the low-energy modes about (2.4), one can alternatively rely entirely on symmetry considerations and, specifically, on the Ward-Takahashi identities. As we will show in the next subsection, such symmetryaware approach permits to obtain the equations of motion for the low-energy modes in a direct way, which is usually more transparent than the standard Lagrangian study of the fluctuations.

\section{A. WARD-TAKAHASHI IDENTITIES AND LOW-ENERGY MODES}

Model (2.2) features a conserved $U(1)$ current given by

$$
J_{\mu}=i\left(\partial_{\mu} \psi^{*} \psi-\psi^{*} \partial_{\mu} \psi\right), \quad \partial^{\mu} J_{\mu}=0,
$$

while the improved energy-momentum tensor is

$$
\begin{aligned}
T_{\mu \nu}= & 2 \partial_{(\mu} \psi^{*} \partial_{\nu)} \psi+\partial_{\mu} \xi \partial_{\nu} \xi-\eta_{\mu \nu} \mathcal{L} \\
& +\frac{1}{3}\left(\eta_{\mu \nu} \partial^{2}-\partial_{\mu} \partial_{\nu}\right)\left(\frac{1}{2} \xi^{2}+|\psi|^{2}\right) .
\end{aligned}
$$

This expression satisfies on-shell the following WardTakahashi identities $^{6}$

$$
T_{[\mu \nu]}=0, \quad \partial^{\mu} T_{\mu \nu}=0, \quad T^{\mu}{ }_{\mu}=0 .
$$

We expand around the vacuum (2.4) by considering the fluctuation parametrization (2.5). Up to linear order in the fields, the $U(1)$ current is given by

$$
J_{\mu} \simeq \sqrt{2} v \partial_{\mu} \vartheta
$$

so that its conservation equation gives the equation of motion for the $U(1)$ NG mode

$$
0=\partial^{\mu} J_{\mu} \simeq \sqrt{2} v \partial^{2} \vartheta
$$

The energy-momentum tensor expanded to linear order is

\footnotetext{
${ }^{6}$ The trace Ward-Takahashi identity requires the improvement introduced in (2.8).
} 


$$
T_{\mu \nu} \simeq \frac{v}{\sqrt{3}}\left(\eta_{\mu \nu} \partial^{2}-\partial_{\mu} \partial_{\nu}\right) \tau,
$$

and the trace Ward-Takahashi identity yields the equation of motion for the dilaton

$$
0=T^{\mu}{ }_{\mu} \simeq \sqrt{3} v \partial^{2} \tau .
$$

From (2.11) and (2.13) we can observe that we recover the two massless modes of (2.6). The Ward-Takahashi computation, however, descends directly from symmetry arguments, being therefore more convenient (and easier) to apply, especially when dealing with models more complicated than (2.2). In particular, this approach allows to identify immediately and without ambiguities the nature of each Goldstone boson, simply by associating every (gapless) mode to the Ward-Takahashi identity that yields its equation of motion.

It is important to stress that the model (2.2) is fine-tuned. Indeed, (classical) scale invariance dictates that the potential should contain only quartic terms in the scalars, but the fact that the potential is a perfect square constitutes a finetuning, specifically considered to the purpose of having a flat direction. The latter is of course a necessary condition for the presence of a low-energy dilaton mode.

The simple argument is as follows. In such a relativistic setup, scale invariance implies the absence of any reference scale in the (effective) Lagrangian. If scale invariance is to be broken spontaneously by a vacuum expectation value (VEV), then the latter must be arbitrary. Hence this VEV parametrizes a noncompact flat direction. Moreover the absence of any reference scale means that the flat direction must also correspond to a vanishing vacuum energy. The particle which corresponds to moving along this flat direction is the dilaton. We conclude that any effective theory that aims at describing spontaneous scale symmetry breaking (among others), must allow for a noncompact flat direction in its potential.

For instance, if we added a generic term preserving scale invariance but breaking the exchange symmetry between $|\psi|$ and $\xi$, namely (without loss of generality)

$$
V=\lambda\left(|\psi|^{2}-\xi^{2}\right)^{2}+\lambda^{\prime}\left(|\psi|^{2}\right)^{2},
$$

the equations extremizing the potential would become

$$
\begin{gathered}
\lambda \psi\left(|\psi|^{2}-\xi^{2}\right)=-\lambda^{\prime}|\psi|^{2} \psi, \\
\lambda \xi\left(|\psi|^{2}-\xi^{2}\right)=0 .
\end{gathered}
$$

Considering $\lambda^{\prime}>0$ for $V$ to be bounded from below, the only solution is $\xi=0=\psi$, i.e., the flat direction is completely lifted, even though scale invariance is respected.

\section{SPONTANEOUS SYMMETRY BREAKING AT FINITE DENSITY}

In this section we depart from the Lorentz-invariant setup discussed above, by introducing a nonzero chemical potential $\mu$ for the charge associated to the global $U(1)$ symmetry. As we will see, we will still be able to identify the dilaton and the $U(1) \mathrm{NG}$, though their dispersion relations will be modified in an interesting way.

We start with a scale-invariant theory defined by the action

$S=\int d^{4} x\left[\partial_{\mu} \psi^{*} \partial^{\mu} \psi+\frac{1}{2} \partial_{\mu} \xi \partial^{\mu} \xi-\lambda\left(|\psi|^{2}-\xi^{2}\right)^{2}-\lambda^{\prime}\left(|\psi|^{2}\right)^{2}\right]$,

whose potential corresponds to the extension already introduced in (2.14). We are going to switch on a chemical potential $\mu$ for the $U(1)$ symmetry. As discussed before, at finite chemical potential, the ground state is no longer determined by the Hamiltonian $H$ but by the effective Hamiltonian $\tilde{H}=H-\mu Q$, where $Q$ is the $U(1)$ charge operator. As we will discuss, this modifies the effective potential of the theory and allows the fields to acquire a nonzero value. Notably, one can recover the zero chemical potential symmetry breaking case described by (2.2) by means of an appropriate limit for both $\mu$ and $\lambda^{\prime}$. The main result of the present section is to show that the dilatonic mode acquires a gap, which depends on $\mu$ and $\lambda^{\prime}$.

A nonzero chemical potential can be implemented by extracting a time-dependent phase from the complex field

$$
\psi=e^{i \mu t} \phi, \quad \psi^{*}=e^{-i \mu t} \phi^{*} .
$$

The equations of motion then read

$$
\begin{aligned}
\partial^{2} \phi+2 i \mu \partial_{0} \phi-\mu^{2} \phi+\partial_{\phi^{*}} V(|\phi|, \xi) & =0, \\
\partial^{2} \xi+\partial_{\xi} V(|\phi|, \xi) & =0,
\end{aligned}
$$

where $V(|\phi|, \xi) \equiv V(|\psi|, \xi)$ is given by (2.14). Note that these equations can equivalently be obtained introducing (3.2) in (3.1), identifying a new effective potential $V_{\phi}(|\phi|, \xi)=V(|\phi|, \xi)-\mu^{2}|\phi|^{2}$ and taking the variation with respect to $\phi^{*}, \xi$. Although $V_{\phi}$ is not the true potential (indeed, the energy density is $E \sim V(|\phi|, \xi)+\mu^{2}|\phi|^{2}$ ), the extrema of $V_{\phi}$ correspond to solutions of the equations of motion of the original action (3.1). We will show in the following that $V_{\phi}$ determines the ground state for the effective Hamiltonian $\tilde{H}$.

\section{A. Effective Hamiltonian and ground state}

In order to determine the effective Hamiltonian and the associated ground state we need to find expressions for the $U(1)$ charge $Q$ and Hamiltonian. We will use the usual 
definitions in terms of the temporal components of the energy-momentum tensor $T_{\mu \nu}$ and $U(1)$ current $J_{\mu}$

$$
H=\int d^{3} x T_{00}, \quad Q=\int d^{3} x J_{0} .
$$

Then, the effective Hamiltonian at finite chemical potential is determined by the temporal component of an effective energy-momentum tensor $t_{\mu \nu}$

$$
\tilde{H}=\int d^{3} x\left(T_{00}-\mu J_{0}\right) \equiv \int d^{3} x t_{00} .
$$

The $U(1)$ current can be written as follows

$$
J_{0}=2 \mu|\phi|^{2}+j_{0}, \quad J_{i}=j_{i},
$$

where

$$
j_{\mu}=i\left(\partial_{\mu} \phi^{*} \phi-\phi^{*} \partial_{\mu} \phi\right) .
$$

Similarly, for the energy-momentum tensor ${ }^{7}$

$$
\begin{gathered}
T_{00}=\mu J_{0}+t_{00}, \\
T_{0 i}=T_{i 0}=\mu J_{i}+t_{0 i}=\mu j_{i}+t_{0 i}, \\
T_{i j}=t_{i j}+\delta_{i j}\left(\mu J_{0}-2 \mu^{2}|\phi|^{2}\right)=t_{i j}+\delta_{i j} \mu j_{0},
\end{gathered}
$$

where

$$
\begin{aligned}
t_{\mu \nu}= & 2 \partial_{(\mu} \phi^{*} \partial_{\nu)} \phi+\partial_{\mu} \xi \partial_{\nu} \xi-\eta_{\mu \nu} \mathcal{L}_{\phi} \\
& +\frac{1}{3}\left(\eta_{\mu \nu} \partial^{2}-\partial_{\mu} \partial_{\nu}\right)\left(\frac{1}{2} \xi^{2}+|\phi|^{2}\right),
\end{aligned}
$$

and

$$
\begin{aligned}
\mathcal{L}_{\phi}= & \partial_{\mu} \phi^{*} \partial^{\mu} \phi+\frac{1}{2} \partial_{\mu} \xi \partial^{\mu} \xi-\lambda\left(|\phi|^{2}-\xi^{2}\right)^{2} \\
& -\lambda^{\prime}\left(|\phi|^{2}\right)^{2}+\mu^{2}|\phi|^{2}
\end{aligned}
$$

Notice that from (3.9) we have that $T_{0 i}=T_{i 0}$ implying that the Ward-Takahashi identities for boost transformations are satisfied, so that the full Lorentz symmetry is still preserved in the presence of a nonvanishing chemical potential.

The effective potential for $\mathcal{L}_{\phi}$ is the one we had identified previously in the equations of motion (3.3)

$$
V_{\phi}=\lambda\left(|\phi|^{2}-\xi^{2}\right)^{2}+\lambda^{\prime}\left(|\phi|^{2}\right)^{2}-\mu^{2}|\phi|^{2}
$$

\footnotetext{
${ }^{7}$ The notations are such that the capital letters $\left(T_{00}\right.$ etc.) refer to the dynamics of $(\psi, \xi)$ (and by extension, of $\phi$ ) dictated by (3.1). The lowercase letters refer instead to the dynamics given by (3.12) which is not the Lagrangian for $(\phi, \xi)$ but it shares the same potential.
}

Since $t_{00}$ determines the effective Hamiltonian (3.5), we see that the ground state will correspond to the minimum of the effective potential. The effective potential has three extrema $^{8}$

$$
\begin{aligned}
& \xi=\phi=0 ; \quad \xi=0,|\phi|^{2}=v^{2}=\frac{\mu^{2}}{2\left(\lambda+\lambda^{\prime}\right)} ; \\
& \xi^{2}=|\phi|^{2}=v^{2}=\frac{\mu^{2}}{2 \lambda^{\prime}} .
\end{aligned}
$$

Out of the three extrema (3.14), the first two are saddle points and only the last is a minimum, which is the true ground state of the system. Note that for the true minimum to exist, and for $V_{\phi}$ to be bounded from below, we need to have $\lambda^{\prime}>0$. In other words, we need to lift the flat direction that we had at $\mu=0$ in order to have a minimum, and symmetry breaking, when $\mu \neq 0$.

We now proceed to investigate the low-energy spectrum around this (degenerate) minimum.

\section{B. Nambu-Goldstone dynamics from Ward-Takahashi identities}

We perturb the fields around the ground state $\xi^{2}=|\phi|^{2}=v^{2}=\frac{\mu^{2}}{2 \lambda^{\prime}}$. We use the same parametrization as in (2.5), though adapted to the field $\phi$

$\phi=e^{i \frac{9}{\sqrt{2 v}}}\left(v e^{\frac{\tau}{\sqrt{3 v}}}+\frac{1}{\sqrt{6}} \rho\right), \quad \xi=v e^{\frac{\tau}{\sqrt{3 v}}}-\frac{2}{\sqrt{6}} \rho$.

As before, the kinetic terms are diagonal and canonically normalized for $\vartheta, \tau$ and $\rho$. We still identify $\vartheta$ as the fluctuation of the phase of the condensate and $\tau$ as a fluctuation of its magnitude, while $\rho$ corresponds to an orthogonal direction of increasing potential energy. For $\mu=0, \vartheta$ and $\tau$ are naturally associated to the $U(1) \mathrm{NG}$ and dilaton, while $\rho$ enters as a Higgs fluctuation. This simple picture is a bit complicated when $\mu \neq 0$, as the would-be Goldstones undergo some mixing and also a nonvanishing gap for one linear combination. We will study this effect in some approximation here and in more detail in the next section.

When the perturbation (3.15) is introduced in the effective potential (3.13) and expanded to quadratic order, one finds no term for $\vartheta$ and the following mass matrix for $(\tau, \rho)$

$$
M=\frac{4 v^{2}}{3}\left(\begin{array}{cc}
2 \lambda^{\prime} & \sqrt{2} \lambda^{\prime} \\
\sqrt{2} \lambda^{\prime} & \lambda^{\prime}+9 \lambda
\end{array}\right) .
$$

In principle both perturbations are massive and mixed, but in the limit $\lambda^{\prime} \ll \lambda$ in which there is an almost flat direction

\footnotetext{
${ }^{8}$ Note that these uniform and static solutions are extrema of the effective potential (3.13), but not of the energy (3.8).
} 
in the original potential (2.14), the mixing becomes very small and there is a large hierarchy between the mass of $\tau$, $m_{\tau}^{2} \sim \lambda^{\prime} v^{2} \sim \mu^{2}$, and the mass of $\rho, m_{\rho}^{2} \sim \lambda v^{2}$. In the following we will assume that we are in this situation, in which case the Higgs fluctuation $\rho$ can be set to zero in the low energy description to a good approximation.

The dynamical equations for the remaining fluctuations can be derived from the Ward-Takahashi identities. When evaluated on shell the $U(1)$ current should be conserved and the trace of the energy momentum tensor should vanish

$$
\partial_{\mu} J^{\mu}=0, \quad T^{\mu}{ }_{\mu}=0 .
$$

This gives two equations, which is sufficient to determine the dynamics of $\vartheta$ and $\tau$. The trace of the energymomentum tensor, to linear order in the fluctuations, is

$$
T_{\mu}^{\mu} \simeq \sqrt{3} v\left(\partial^{2} \tau+\frac{4}{3} \mu^{2} \tau-2 \sqrt{\frac{2}{3}} \mu \partial_{0} \vartheta\right),
$$

whereas the divergence of the current is

$$
\partial^{\mu} J_{\mu} \simeq \sqrt{2} v\left(\partial^{2} \vartheta+2 \sqrt{\frac{2}{3}} \mu \partial_{0} \tau\right) .
$$

This translates into the set of coupled equations

$$
\begin{aligned}
\partial^{2} \tau+\frac{4}{3} \mu^{2} \tau-2 \sqrt{\frac{2}{3}} \mu \partial_{0} \vartheta & \simeq 0, \\
\partial^{2} \vartheta+2 \sqrt{\frac{2}{3}} \mu \partial_{0} \tau & \simeq 0 .
\end{aligned}
$$

As suggested by the general analysis in the introduction, the chemical potential introduces a mixing between the $U(1)$ NG and the dilaton. The equations can be diagonalized using expansions in Fourier modes

$$
\begin{aligned}
& \tau\left(x^{0}, \mathbf{x}\right)=\int \frac{d \omega d^{3} q}{(2 \pi)^{4}} e^{-i \omega x^{0}+i \mathbf{q} \cdot \mathbf{x}} \tilde{\tau}(\omega, \mathbf{q}), \\
& \vartheta\left(x^{0}, \mathbf{x}\right)=\int \frac{d \omega d^{3} q}{(2 \pi)^{4}} e^{-i \omega x^{0}+i \mathbf{q} \cdot \mathbf{x}} \tilde{\vartheta}(\omega, \mathbf{q}) .
\end{aligned}
$$

Expanding at low momentum $q^{2} / \mu^{2} \ll 1$, the equations have solutions when the modes satisfy the dispersion relations

$$
\omega^{2} \simeq \frac{q^{2}}{3}, \quad \omega^{2} \simeq 4 \mu^{2}+\frac{5}{3} q^{2}
$$

Therefore, there is a gapless mode $\pi$ and a gapped mode $\sigma$, which at low momentum correspond respectively to the combinations

$$
\begin{aligned}
& \tilde{\pi} \simeq \tilde{\vartheta}-i \operatorname{sign}(\omega / q) \frac{q}{\sqrt{2} \mu} \tilde{\tau}, \\
& \tilde{\sigma} \simeq \tilde{\tau}-i \sqrt{\frac{2}{3}} \operatorname{sign}(\omega / \mu)\left(1+\frac{q^{2}}{24 \mu^{2}}\right) \tilde{\vartheta} .
\end{aligned}
$$

A few comments are in order. In the first place, the dispersion relation of $\pi$ in (3.22) is such that it moves at the speed of sound as fixed by conformal invariance $c_{s}^{2}=1 / 3$, i.e., it can be identified as a conformal superfluid phonon, while $\sigma$ is the gapped dilaton. This identification is consistent with an effective field theory approach, see e.g., [4]. Note that the mixing is necessary for this to happen, otherwise the phonon would move at the speed of light due to relativistic invariance of the rest of the terms. The second observation is that the gap of $\sigma$ is fixed by the chemical potential $m_{\sigma}=2 \mu$, and independent of the couplings $\lambda$ and $\lambda^{\prime}$ in this approximation. This is very reminiscent of the massive Goldstone bosons appearing when internal symmetries are spontaneously broken in the presence of a chemical potential. A last observation is that because of the mixing, it is no longer true that each Ward-Takahashi identity is tied to one specific mode. Indeed reexpressing $\tau$ and $\vartheta$ in terms of $\pi$ and $\sigma$, one can easily see that both fields appear in both equations (3.20).

\section{Exact dispersion relations}

The results obtained from the Ward-Takahashi identities are easy to interpret physically but we had to introduce several approximations to derive them, in particular we used the hierarchy between the masses of the Higgs fluctuation and the dilaton to freeze out the first. In order to go beyond this approximation we need to include the Higgs mode in the analysis, whose dynamics is not captured by the Ward-Takahashi identities. This can be more simply done using the effective Lagrangian.

Consider again the vacuum $\xi^{2}=|\phi|^{2}=v^{2}=\frac{\mu^{2}}{2 \lambda^{\prime}}$ and the fluctuations (3.15) around it. The quadratic Lagrangian for the fluctuations is

$$
\begin{aligned}
\mathcal{L}_{\text {quad }}= & \frac{1}{2} \partial_{\mu} \rho \partial^{\mu} \rho+\frac{1}{2} \partial_{\mu} \vartheta \partial^{\mu} \vartheta+\frac{1}{2} \partial_{\mu} \tau \partial^{\mu} \tau \\
& +2 \sqrt{\frac{2}{3}} \mu \tau \partial_{t} \theta+\frac{2}{\sqrt{3}} \mu \rho \partial_{t} \theta-\frac{2}{3} \sqrt{2} \mu^{2} \tau \rho \\
& -\frac{2}{3} \mu^{2} \tau^{2}-\mu^{2} \frac{9 \lambda+\lambda^{\prime}}{3 \lambda^{\prime}} \rho^{2} .
\end{aligned}
$$

By going to Fourier space we get

$$
\mathcal{L}_{\text {quad }}=\frac{1}{2} y^{T}(-\omega,-q) \cdot M(\omega, q) \cdot y(\omega, q), \quad y=(\vartheta, \rho, \tau),
$$

where 


$$
M=\left(\begin{array}{ccc}
\omega^{2}-q^{2} & i \frac{2}{\sqrt{3}} \mu \omega & i \frac{2 \sqrt{2}}{\sqrt{3}} \mu \omega \\
-i \frac{2}{\sqrt{3}} \mu \omega & \omega^{2}-q^{2}-\frac{2\left(9 \lambda+\lambda^{\prime}\right)}{3 \lambda^{\prime}} \mu^{2} & -\frac{2 \sqrt{2}}{3} \mu^{2} \\
-i \frac{2 \sqrt{2}}{\sqrt{3}} \mu \omega & -\frac{2 \sqrt{2}}{3} \mu^{2} & \omega^{2}-q^{2}-\frac{4}{3} \mu^{2}
\end{array}\right) .
$$

Studying the zeros of the determinant of $M$, one finds one massless mode, the $U(1)$ NG boson, and two gapped modes:

$$
\begin{aligned}
\left.\omega_{1}^{2}\right|_{q=0} & =0 \\
\left.\omega_{2,3}^{2}\right|_{q=0} & =\frac{3 \mu^{2}}{\lambda^{\prime}}\left(\lambda+\lambda^{\prime} \pm \sqrt{\lambda^{2}-\frac{2}{3} \lambda \lambda^{\prime}+\lambda^{\prime 2}}\right) .
\end{aligned}
$$

Expanding for low momentum $q$ and for $\lambda^{\prime} \ll \lambda$, we get

$$
\begin{gathered}
\omega_{1}^{2} \simeq \frac{1}{3} q^{2} \\
\omega_{2}^{2} \simeq 6 \mu^{2} \frac{\lambda}{\lambda^{\prime}}\left(1+\frac{\lambda^{\prime}}{3 \lambda}\right)+\left(1+\frac{2 \lambda^{\prime}}{9 \lambda}\right) q^{2}, \\
\omega_{3}^{2} \simeq 4 \mu^{2}\left(1-\frac{\lambda^{\prime}}{3 \lambda}\right)+\left(\frac{5}{3}-\frac{2 \lambda^{\prime}}{9 \lambda}\right) q^{2} .
\end{gathered}
$$

Comparing with the dispersion relations in (3.22), we observe that the speed of the phonon is not modified by corrections depending on $\lambda^{\prime}$, while the mass of the gapped dilaton is corrected, though mildly. Indeed, contrary to massive NG bosons associated to internal symmetries, the mass of the gapped dilaton is not protected by the symmetry.

For $\lambda^{\prime} \ll \lambda, \omega_{1}^{2}$ and $\omega_{3}^{2}$ reduce to the dispersion relations obtained in (3.22) from the study of the Ward-Takahashi identities, and we have a hierarchy between the two massive modes. Furthermore, in the limit

$$
\mu \rightarrow 0, \quad \lambda^{\prime} \rightarrow 0 \quad \text { with } \quad \frac{\mu^{2}}{2 \lambda^{\prime}} \rightarrow v^{2},
$$

we recover the masses (2.6) of the relativistic model (2.2)

$$
\begin{aligned}
& \left.\omega_{1}^{2}\right|_{q=0}=0, \\
& \left.\omega_{2}^{2}\right|_{q=0}=12 v^{2} \lambda, \\
& \left.\omega_{3}^{2}\right|_{q=0}=0,
\end{aligned}
$$

and $\omega_{3}$ describes the massless dilaton. This suggests a connection between the corrections to the mass of the gapped dilaton at finite chemical potential and the lack of a flat direction in the potential at zero chemical potential. The masses of gapped NGs might be protected only if there are flat directions associated to them, of course this will always be the case for internal symmetries.

\section{BOOSTED SUPERFLUID}

Since the chemical potential breaks Lorentz invariance, it is interesting to study the effect on the NG modes when the superfluid is set on motion relative to the frame determined by the effective Hamiltonian induced by the chemical potential, that one can identify as the "laboratory" frame. We consider again (2.2) and introduce both a chemical potential and a superfluid velocity

$$
\psi=e^{i \mu_{0} u_{\mu} x^{\mu}} \phi, \quad \psi^{*}=e^{-i \mu_{0} u_{\mu} x^{\mu}} \phi^{*} .
$$

Where $u_{\mu}=\gamma(1,-\vec{\beta}), \gamma=1 / \sqrt{1-|\beta|^{2}}$ is a timelike fourvelocity $u_{\mu} u^{\mu}=+1$. The chemical potential is $\mu=\gamma \mu_{0}$, and the time direction in the laboratory frame is $x^{0}$. The background plane wave (4.1) is the same as (3.2) seen by a boosted observer, compared to the laboratory frame. Since (2.2) is Lorentz invariant, the dispersion relations for the gapless low-energy modes can be obtained by boosting those obtained from (3.1) (i.e., the case with just a chemical potential). For the sake of providing an explicit check, we repeat the exercise of computing them directly through the Ward-Takahashi identities and through the perturbative Lagrangian approach.

\section{A. Effective Hamiltonian and ground state}

We proceed in a similar fashion to the case of zero velocity. The Hamiltonian and the charge are still determined by the energy-momentum tensor and the current as in (3.4), and the effective Hamiltonian at nonzero chemical potential by (3.5). Because of the boost, the expressions for the current and the energy-momentum tensor are slightly modified.

$$
\begin{aligned}
J_{\mu} & =2 \mu_{0}|\phi|^{2} u_{\mu}+j_{\mu}, \\
T_{\mu \nu} & =2 \mu_{0}^{2} u_{\mu} u_{\nu}|\phi|^{2}+\mu_{0}\left(u_{\mu} j_{\nu}+u_{\nu} j_{\mu}\right)-\eta_{\mu \nu} \mu_{0} u^{\alpha} j_{\alpha}+t_{\mu \nu}\left(\mu_{0}\right) .
\end{aligned}
$$

Where $j^{\mu}$ and $t_{\mu \nu}$ take the same form as before (3.7) and (3.11), replacing $\mu$ by $\mu_{0}$. Recalling that the chemical potential is $\mu=\mu_{0} u_{0}=\mu_{0} \gamma$, the effective Hamiltonian is

$$
H-\mu Q=\int d^{3} x\left(T_{00}-\mu J_{0}\right)=\int d^{3} x\left(t_{00}\left(\mu_{0}\right)-\mu \vec{\beta} \cdot \vec{j}\right) .
$$

Since $j_{i}$ vanishes for constant $\phi$, the extrema of the effective potential are the same as before (3.14) replacing $\mu$ by the effective chemical potential in the rest frame of the fluid $\mu_{0}$. The ground state is thus $\xi^{2}=|\phi|^{2}=v_{0}^{2}=\frac{\mu_{0}^{2}}{2 \lambda^{\prime}}$. 


\section{B. Nambu-Goldstone dynamics from Ward-Takahashi identities}

We can use the same parametrization for perturbations of the ground state as in (3.15), replacing $v$ by $v_{0}$. The same considerations about the mass hierarchy of $\tau$ and $\rho$ apply, so in this analysis we will assume $\lambda^{\prime} \ll \lambda$ and freeze $\rho$. The dynamics of the low energy modes are determined by the conservation equations for the current and the energymomentum tensor. For the boosted superfluid they take the form

$$
\begin{aligned}
\partial^{\mu} J_{\mu} & =2 \mu\left(\partial_{0}+\vec{\beta} \cdot \vec{\nabla}\right)|\phi|^{2}+\partial^{\mu} j_{\mu}, \\
T^{\mu}{ }_{\mu} & =2 \mu_{0}^{2}|\phi|^{2}-2 \mu_{0} u^{\mu} j_{\mu}+t^{\mu}{ }_{\mu}\left(\mu_{0}\right) \\
& =2 \mu_{0}^{2}|\phi|^{2}-2 \mu\left(j_{0}+\vec{\beta} \cdot \vec{j}\right)+t^{\mu}{ }_{\mu}\left(\mu_{0}\right) .
\end{aligned}
$$

Therefore, we should just replace the terms with a single time derivative by the material derivative $\mu \partial_{0} \rightarrow \mu D_{0}=$ $\mu\left(\partial_{0}+\vec{\beta} \cdot \vec{\nabla}\right)$ and otherwise change $\mu$ by the effective $\mu_{0}$ :

$$
\begin{aligned}
\partial^{\mu} J_{\mu} & \simeq \sqrt{2} v_{0}\left(\partial^{2} \vartheta+2 \sqrt{\frac{2}{3}} \mu D_{0} \tau\right) \\
T_{\mu}^{\mu} & \simeq \sqrt{3} v_{0}\left(\partial^{2} \tau+\frac{4}{3} \mu_{0}^{2} \tau-2 \sqrt{\frac{2}{3}} \mu D_{0} \vartheta\right) .
\end{aligned}
$$

From this, we obtain the equations

$$
\begin{aligned}
\partial^{2} \tau+\frac{4}{3} \mu_{0}^{2} \tau-2 \sqrt{\frac{2}{3}} \mu D_{0} \vartheta & \simeq 0, \\
\partial^{2} \vartheta+2 \sqrt{\frac{2}{3}} \mu D_{0} \tau & \simeq 0 .
\end{aligned}
$$

The dispersion relation for the gapless mode can be more easily found by noting that $\mu=\gamma \mu_{0}$ and using comoving coordinates. Taking $\vec{\beta}$ parallel to the $x^{3}$ direction, we introduce

$$
\begin{aligned}
& x^{0}=\gamma\left(x_{\beta}^{0}+\beta x_{\beta}^{3}\right), \quad x^{3}=\gamma\left(x_{\beta}^{3}+\beta x_{\beta}^{0}\right), \\
& x^{1}=x_{\beta}^{1}, \quad x^{2}=x_{\beta}^{2} .
\end{aligned}
$$

Then

$\frac{\partial}{\partial x_{\beta}^{0}}=\gamma\left(\partial_{0}+\beta \partial_{3}\right), \quad \frac{\partial}{\partial x_{\beta}^{3}}=\gamma\left(\partial_{3}+\beta \partial_{0}\right), \quad \partial^{2}=\partial_{\beta}^{2}$.

The equations become

$$
\begin{aligned}
\partial_{\beta}^{2} \tau+\frac{4}{3} \mu_{0}^{2} \tau-2 \sqrt{\frac{2}{3}} \mu_{0} \partial_{x_{\beta}^{0}} \vartheta & \simeq 0, \\
\partial_{\beta}^{2} \vartheta+2 \sqrt{\frac{2}{3}} \mu_{0} \partial_{x_{\beta}^{0}} \tau & \simeq 0 .
\end{aligned}
$$

These are the same as before (3.20), replacing $\mu$ by $\mu_{0}$. We introduce an expansion of the modes in the rest frame in plane waves

$$
\begin{aligned}
\tau\left(x_{\beta}^{0}, \mathbf{x}_{\beta}\right) & =\int \frac{d \omega_{\beta} d^{3} q_{\beta}}{(2 \pi)^{4}} e^{-i \omega_{\beta} x_{\beta}^{0}+i \mathbf{q}_{\beta} \cdot \mathbf{x}_{\beta}} \tilde{\tau}\left(\omega_{\beta}, \mathbf{q}_{\beta}\right), \\
\vartheta\left(x_{\beta}^{0}, \mathbf{x}_{\beta}\right) & =\int \frac{d \omega_{\beta} d^{3} q_{\beta}}{(2 \pi)^{4}} e^{-i \omega_{\beta} x_{\beta}^{0}+i \mathbf{q}_{\beta} \cdot \mathbf{x}_{\beta}} \tilde{\vartheta}\left(\omega_{\beta}, \mathbf{q}_{\beta}\right) .
\end{aligned}
$$

We recover the expected low momentum dispersion relations in the rest frame

$$
\omega_{\beta}^{2} \simeq c_{s}^{2} q_{\beta}^{2}, \quad \omega_{\beta}^{2} \simeq 4 \mu_{0}^{2}+\frac{5}{3} q_{\beta}^{2},
$$

where $c_{s}^{2}=1 / 3$ is the speed of sound of the scale invariant theory. These expressions can be translated to frequency and momentum in the laboratory frame using that

$$
\begin{aligned}
\omega & =\gamma\left(\omega_{\beta}+\beta q_{\beta 3}\right), \quad q_{3}=\gamma\left(q_{\beta 3}+\beta \omega_{\beta}\right), \\
q_{1} & =q_{\beta 1}, \quad q_{2}=q_{\beta 1} .
\end{aligned}
$$

Note that the dispersion relations (4.11) are valid for low momentum in the rest frame of the fluid $\left|q_{\beta}\right| \ll\left|\mu_{0}\right|$. For the gapless modes they can be matched with a low momentum expansion in the laboratory frame $|q| \ll|\mu|$, however for the gapped modes this is not possible, as for generic $\beta$, $q_{3} \sim \omega_{\beta} \sim \mu$. Therefore, finding the dispersion relations of the gapped modes at low momentum in the laboratory frame requires solving (4.6) directly.

We classify the dispersion relations of the gapless modes taking as reference the direction of the superfluid velocity in the laboratory frame. The dispersion relations for the longitudinal modes is

$$
\omega_{\|}= \pm \frac{c_{s} \pm \beta}{1 \pm \beta c_{s}} q_{3}, \quad q_{1}=q_{2}=0
$$

while the dispersion relation for the transverse modes is

$$
\omega_{\perp}^{2}=c_{s}^{2} \frac{q_{1}^{2}+q_{2}^{2}}{\gamma^{2}\left(1-\beta^{2} c_{s}^{2}\right)}, \quad q_{3}=0 .
$$

These expressions agree with the ones obtained by relativistic addition of velocities. Note that for $|\beta|>c_{s}$ both (positive frequency) longitudinal modes (4.13) propagate in the same direction as the superfluid velocity. This is the reason why we expressed linearly the dispersion relations. 
For the gapped modes the low momentum dispersion relations are

$$
\begin{aligned}
\omega_{\|} & =2 \sqrt{1-\beta^{2} c_{s}^{2}} \mu-\frac{2}{3} \frac{\beta q_{3}}{1-\beta^{2} c_{s}^{2}}+\frac{5+\beta^{2} c_{s}^{2}}{12 \gamma^{2}\left(1-\beta^{2} c_{s}^{2}\right)^{5 / 2}} \frac{q_{3}^{2}}{\mu}, \\
q_{1} & =q_{2}=0, \\
\omega_{\perp}^{2} & =4\left(1-\beta^{2} c_{s}^{2}\right)^{2} \mu^{2}+\frac{5-\beta^{2}}{3\left(1-\beta^{2} c_{s}^{2}\right)}\left(q_{1}^{2}+q_{2}^{2}\right), \quad q_{3}=0,
\end{aligned}
$$

where again the longitudinal dispersion relation is expressed linearly. The gap is reduced by the superfluid velocity, but in this approximation remains finite even in the limit $\beta \rightarrow 1$, where the condensate vanishes (i.e., at fixed $\mu$ ). Note also that, at leading order for momenta in the same direction of the flow, the frequency is reduced.

\section{Exact dispersion relations}

We now study the effects of including the Higgs fluctuation $\rho$, and the corrections for finite $\lambda^{\prime} / \lambda$. We thus resort to expanding the full Lagrangian. According to (4.1), we switch on a chemical potential $\mu=\mu_{0} \gamma$ and a background wave vector $k_{3}=\mu_{0} \gamma \beta$. The effective potential is now:

$$
V=\lambda\left(|\phi|^{2}-\xi^{2}\right)^{2}+\lambda^{\prime}|\phi|^{4}-\left(\mu^{2}-k_{3}^{2}\right)|\phi|^{2},
$$

For stationary solutions, the situation is not very different from the case with just $\mu$, in fact one just needs to replace $\mu^{2}$ by $\left(\mu^{2}-k_{3}^{2}\right)=\mu_{0}^{2}$ in (3.13). Therefore, we consider the solution

$$
\xi^{2}=|\phi|^{2}, \quad|\phi|^{2}=\frac{\mu_{0}^{2}}{2 \lambda^{\prime}} .
$$

The fluctuation around (4.17) are still given by (3.15) where however $v=v_{0}=\frac{\mu_{0}}{\sqrt{2 \lambda^{\prime}}}$. Writing $k_{3}=\beta \mu$, the quadratic Lagrangian for the fluctuations is

$$
\begin{aligned}
\mathcal{L}_{\text {quad }}= & \frac{1}{2} \partial_{\mu} \rho \partial^{\mu} \rho+\frac{1}{2} \partial_{\mu} \vartheta \partial^{\mu} \vartheta+\frac{1}{2} \partial_{\mu} \tau \partial^{\mu} \tau \\
& +2 \sqrt{\frac{2}{3}} \mu \tau\left(\partial_{t}+\beta \partial_{3}\right) \theta+\frac{2}{\sqrt{3}} \mu \rho\left(\partial_{t}+\beta \partial_{3}\right) \theta \\
& -\frac{2}{3} \sqrt{2} \mu_{0}^{2} \rho \tau-\frac{2}{3} \mu_{0}^{2} \tau^{2}-\mu_{0}^{2} \frac{9 \lambda+\lambda^{\prime}}{3 \lambda^{\prime}} \rho^{2} .
\end{aligned}
$$

In analogy to (3.25) and (3.26), by going to Fourier space we get the kinetic matrix:

$$
\left(\begin{array}{ccc}
\omega^{2}-q^{2} & i \frac{2}{\sqrt{3}} \mu\left(\omega-\beta q_{3}\right) & i \frac{2 \sqrt{2}}{\sqrt{3}} \mu\left(\omega-\beta q_{3}\right) \\
-i \frac{2}{\sqrt{3}} \mu\left(\omega-\beta q_{3}\right) & \omega^{2}-q^{2}-\frac{2\left(9 \lambda+\lambda^{\prime}\right)}{3 \lambda^{\prime}} \mu_{0}^{2} & -\frac{2 \sqrt{2}}{3} \mu_{0}^{2} \\
-i \frac{2 \sqrt{2}}{\sqrt{3}} \mu\left(\omega-\beta q_{3}\right) & -\frac{2 \sqrt{2}}{3} \mu_{0}^{2} & \omega^{2}-q^{2}-\frac{4}{3} \mu_{0}^{2}
\end{array}\right)
$$

From the determinant of (4.19), one can find the exact dispersion relations. First of all, setting the momenta $q=0$ one finds that there is a massless mode corresponding to the $U(1)$ NG boson and two gapped modes:

$$
\left.\omega_{1}^{2}\right|_{q=0}=0,\left.\quad \omega_{2,3}^{2}\right|_{q=0}=\frac{3}{\lambda^{\prime}}\left[\lambda \mu_{0}^{2}+\lambda^{\prime} \mu^{2}\left(1-c_{s}^{2} \beta^{2}\right) \pm \sqrt{\lambda^{2} \mu_{0}^{4}-\frac{2}{3} \lambda \mu_{0}^{2} \lambda^{\prime} \mu^{2}\left(1-c_{s}^{2} \beta^{2}\right)+\lambda^{2} \mu^{4}\left(1-c_{s}^{2} \beta^{2}\right)^{2}}\right],
$$

where $c_{s}=1 / 3$ as before.

Now, expanding at low frequencies and momenta, one can extract analytically the dispersion relation for the $U(1)$ NG mode:

$$
\omega_{1}=\frac{c_{s}}{1-c_{s}^{2} \beta^{2}}\left(2 c_{s} \beta q_{3} \pm \sqrt{\left(1-\beta^{2}\right)^{2} q_{3}^{2}+\left(1-\beta^{2}\right)\left(1-c_{s}^{2} \beta^{2}\right)\left(q_{1}^{2}+q_{2}^{2}\right)}\right)
$$

Notice that the above expression is independent of the ratio $\lambda^{\prime} / \lambda$. Indeed one can check that in the longitudinal and transverse case, it reproduces correctly the expressions (4.13) and (4.14), respectively.

For the massive modes, one has to expand the frequencies around the respective gaps. To first order in momenta and in $\lambda^{\prime} / \lambda$, the dispersion relations for the gapped dilaton are: 


$$
\begin{aligned}
& \omega_{\|}^{\text {(gapped) }}=2 \mu \sqrt{1-c_{s}^{2} \beta^{2}}\left[1-\frac{\lambda^{\prime}}{6 \lambda} \frac{1-c_{s}^{2} \beta^{2}}{1-\beta^{2}}\right]-\frac{2 \beta}{3\left(1-c_{s}^{2} \beta^{2}\right)}\left[1-\frac{\lambda^{\prime}}{3 \lambda} \frac{1-c_{s}^{2} \beta^{2}}{1-\beta^{2}}\right] q_{3}+\ldots \\
& \omega_{\perp}^{\text {(gapped) }}=2 \mu \sqrt{1-c_{s}^{2} \beta^{2}}\left[1-\frac{\lambda^{\prime}}{6 \lambda} \frac{1-c_{s}^{2} \beta^{2}}{1-\beta^{2}}\right]+\frac{1}{12 \sqrt{1-c_{s}^{2} \beta^{2}}}\left[\frac{5-\beta^{2}}{1-c_{s}^{2} \beta^{2}}+\frac{\lambda^{\prime}}{6 \lambda}\right] \frac{q_{1}^{2}+q_{2}^{2}}{\mu}+\ldots
\end{aligned}
$$

For $\lambda^{\prime} / \lambda=0$ they agree with the dispersion relations obtained from the Ward-Takahashi identities (4.15). Again, we observe that the gap receives corrections in $\lambda^{\prime} / \lambda$, so it is not protected by the symmetry.

Finally, we present the dispersion relations of the Higgs mode, to the same order:

$$
\begin{aligned}
& \omega_{\|}^{\text {(heavy) }}=\sqrt{\frac{6 \lambda}{\lambda^{\prime}}} \sqrt{1-\beta^{2}} \mu\left[1+\frac{\lambda^{\prime}}{6 \lambda} \frac{1-c_{s}^{2} \beta^{2}}{1-\beta^{2}}\right]-\frac{2 \lambda^{\prime}}{9 \lambda} \frac{\beta}{1-\beta^{2}} q_{3}+\ldots \\
& \omega_{\perp}^{\text {(heavy) }}=\sqrt{\frac{6 \lambda}{\lambda^{\prime}}} \sqrt{1-\beta^{2}} \mu\left[1+\frac{\lambda^{\prime}}{6 \lambda} \frac{1-c_{s}^{2} \beta^{2}}{1-\beta^{2}}\right]+\frac{1}{2 \sqrt{1-\beta^{2}}} \sqrt{\frac{\lambda^{\prime}}{6 \lambda}} \frac{q_{1}^{2}+q_{2}^{2}}{\mu}+\ldots
\end{aligned}
$$

Note that the $\beta \rightarrow 1$ limit at fixed $\mu$ seems to be ill defined. However this is an artifact of the expansion. For instance, inspecting (4.20) and noticing that in this limit $\mu_{0} \rightarrow 0$, we find that the gap of the dilaton actually goes to zero, while the gap of the Higgs mode stays finite, but scales with $\mu$, which might be slightly nonintuitive (recall that in this limit there is no condensate).

\section{SUMMARY AND DISCUSSION}

The two main highlights of the present paper are these:

(1) The analysis of the low-energy mode associated to spontaneously broken scale symmetry and the characterization of how its zero-temperature gap depends on the finite density.

(2) The description of a generic method based on WardTakahashi identities alone to study the low-energy modes of an effective field theory.

The analysis pursued in the present paper indicates that the spontaneous breaking of the scale symmetry at zero temperature gives rise to a light dilatonic mode whose gap is directly proportional to the chemical potential. This generic expectation can be relevant for the low-energy content of zero-temperature systems where the chemical potential, too, is small with respect to the UV cutoff of the effective description (related to some other physical scale such as an external magnetic field [28]).

Ward-Takahashi identities in quantum field theory are known to be a key tool for the study of symmetries, either when these are preserved or broken, and even when the breaking is explicit [29,30]. The present paper stresses that Ward-Takahashi identities alone provide a sufficient framework to study the dispersion relations of the low-energy modes of an effective field theory, providing an alternative - generally simpler-approach than the direct fluctuation analysis at the level of the Lagrangian. The method is generic, but we applied it to the specific study of scale symmetry breaking to the purpose of elucidating the characteristics of the resulting low-energy dynamics. It would be interesting to look for a gapped dilaton in a strongly coupled theory, by means of the holographic duality. This might be achieved by combining holographic models with a gapless dilaton in Poincaré invariant vacua [31,32] (see also [33]) and models with type II and gapped NG modes [34,35].

We first examined a relativistic field-theory model (2.2) in four spacetime dimensions where scale symmetry and a global $U(1)$ symmetry are concomitantly and spontaneously broken. The scale-invariant potential must have two flat directions which translate into two gapless NG modes, the dilaton and the $U(1) \mathrm{NG}$ both relativistic and both propagating at the speed of light, (2.11) and (2.13).

In order to realize the same symmetry-breaking pattern at finite density, the model must be stabilized by means of an extra scale-invariant term (3.1) which lifts the dilatonic flat direction without affecting the spontaneous nature of the breaking. The resulting low-energy modes are nonetheless altered: the $U(1) \mathrm{NG}$ remains gapless but propagates at the conformal speed of sound, like a superfluid phonon; the dilaton acquires a gap of the order of the chemical potential $\mu$ whose value is however not protected by symmetry (3.22). The dilaton is light compared to other gapped modes only when the coefficient of the term that lifts the flat direction (3.1) is tuned to be very small, in that case we observe that the dilaton gap becomes independent of the couplings.

Our results at nonzero density belong to the line of research on gapped NG modes [15,19-21,36]. In this context, a natural future perspective is to embed the present analysis into a systematic Maurer-Cartan effective framework, thus assessing its universality and possible generalizations.

One interesting field of applications is provided by condensed matter. The presence of a wide critical 
region in the phase diagram is a characteristic shared by many-generally strongly correlated-systems, among which the cuprates. The critical phase is associated to interesting phenomena like bad and strange metallicity and non-Fermi liquid behavior [37]. It is also often conjectured to lie at the basis of the mechanism for high-temperature superconductivity, see for instance [38].

The defining property of such critical region is the validity of simple scaling rules whose origin, however, can involve complicated and often elusive dynamics related to the presence of a quantum critical point [39-41] or, more generally, to the presence of a scaling sector [38,42]. This is sometimes referred to as generic scale invariance [43] and can be assumed among the defining symmetries of an effective description.

Another paradigmatic example is provided by cold atoms at unitarity, where there is an emergent nonrelativistic conformal symmetry [44], known as Schroedinger symmetry. Gapped NG modes are known to appear when the Hamiltonian is deformed by some of the symmetry generators of the Schroedinger algebra [45]. An extension of our analysis, along the lines of [23], to systems with Galilean rather than Lorentz invariance would be quite interesting.

As another remark, still related to condensed matter but in the context of standard metals, it is relevant to mention that the low-energy modes of our analysis would not destabilize a Landau-Fermi liquid coexisting with them. This can be appreciated by means of an extension of the results of [46] to dilatations, a symmetry which does not commute with either spatial or temporal translations: one can show that the linear interaction term between the fermionic quasiparticles and, respectively, the $U(1) \mathrm{NG}$ and the dilaton are both vanishing.

The model adopted here allows for generalizations in which the $U(1)$ symmetry is coupled to translations and the symmetry-breaking preserves only a linear combination of the two [47]. This would realize a spatial version of the pattern described above when $\mu \neq 0$ and only a diagonal component of the product of internal $U(1)$ and time translations was preserved. Such breakings are referred to as homogeneous because they do not yield any spacetime modulation of the energy density, ${ }^{9}$ they however provide acoustic phonon modes. It is an interesting open question to study whether and how these phonons would coexist with a dilatonic mode [49]. The relevance of the question is threefold: it relates to the counting problem of NG modes for spacetime symmetries $[9,16]$; it concerns condensed matter systems where a critical scaling and the breaking of translations are intertwined ${ }^{10}$; it provides insight regarding holographic models where scaling and translation symmetries are broken together [51-54]. ${ }^{11}$

\section{ACKNOWLEDGMENTS}

We are grateful to Tomas Brauner for useful comments. R. A. and C. H. want to thank Nordita for their hospitality during the program "Effective Theories of Quantum Phases of Matter." R. A. and D. N. acknowledge support by IISNBelgium (convention 4.4503.15) and by the F. R. S.-FNRS under the "Excellence of Science" EOS be.h Project No. 30820817. R. A. is supported as a Research Director of the F. R. S.-FNRS (Belgium). C. H. has been partially supported by the Spanish Ministerio de Ciencia, Innovacion y Universidades Grant No. PGC2018096894-B-100 and by the Principado de Asturias through Grant No. GRUPIN-IDI/2018/000174.

\footnotetext{
${ }^{9}$ An example of inhomogeneous breaking of spatial translations in field theory was studied in [48]. Studying the cubic polynomial in $\omega^{2}$ associated to (3.26), one can exclude the presence of complex solutions. Similarly, a numerical study of (4.19) showed no hints of finite-momentum instabilities. Thus, for the purpose of studying translation symmetry breaking, the models introduced in the main text need to be enriched and generalized.

${ }^{10}$ Such as in the region of the phase diagram of cuprates overlapping with the critical, strange metal phase and the socalled pseudogap phase $[37,50]$.

${ }^{11}$ Merging the last two points, there is a current in the literature addressing the critical breaking of translations relevant for the study of strongly correlated electron systems (specifically strange and bad metals), see for instance [55-62].
}

[1] S. Hellerman, D. Orlando, S. Reffert, and M. Watanabe, On the CFT operator spectrum at large global charge, J. High Energy Phys. 12 (2015) 071.

[2] L. Alvarez-Gaume, O. Loukas, D. Orlando, and S. Reffert, Compensating strong coupling with large charge, J. High Energy Phys. 04 (2017) 059.

[3] A. Monin, D. Pirtskhalava, R. Rattazzi, and F. K. Seibold, Semiclassics, Goldstone bosons and CFT data, J. High Energy Phys. 06 (2017) 011.
[4] D. Orlando, S. Reffert, and F. Sannino, Near-conformal dynamics at large charge, Phys. Rev. D 101, 065018 (2020).

[5] Y. Nambu, Quasiparticles and gauge invariance in the theory of superconductivity, Phys. Rev. 117, 648 (1960).

[6] J. Goldstone, Field theories with superconductor solutions, Nuovo Cimento 19, 154 (1961).

[7] J. Goldstone, A. Salam, and S. Weinberg, Broken symmetries, Phys. Rev. 127, 965 (1962). 
[8] E. Ivanov and V. Ogievetsky, The inverse Higgs phenomenon in nonlinear realizations, Teor. Mat. Fiz. 25, 164 (1975).

[9] I. Low and A. V. Manohar, Spontaneously Broken SpaceTime Symmetries and Goldstone's Theorem, Phys. Rev. Lett. 88, 101602 (2002).

[10] H. Watanabe and H. Murayama, Redundancies in NambuGoldstone Bosons, Phys. Rev. Lett. 110, 181601 (2013).

[11] T. Brauner and H. Watanabe, Spontaneous breaking of spacetime symmetries and the inverse Higgs effect, Phys. Rev. D 89, 085004 (2014).

[12] H. Nielsen and S. Chadha, On how to count goldstone bosons, Nucl. Phys. B105, 445 (1976).

[13] H. Watanabe and T. Brauner, On the number of NambuGoldstone bosons and its relation to charge densities, Phys. Rev. D 84, 125013 (2011).

[14] H. Watanabe and H. Murayama, Unified Description of Nambu-Goldstone Bosons without Lorentz Invariance, Phys. Rev. Lett. 108, 251602 (2012).

[15] A. Kapustin, Remarks on nonrelativistic Goldstone bosons, arXiv:1207.0457.

[16] H. Watanabe, Counting rules of Nambu-Goldstone modes, Annu. Rev. Condens. Matter Phys. 11, 169 (2020).

[17] V. Miransky and I. Shovkovy, Spontaneous Symmetry Breaking with Abnormal number of Nambu-Goldstone Bosons and Kaon Condensate, Phys. Rev. Lett. 88, 111601 (2002).

[18] T. Schäfer, D. Son, M. A. Stephanov, D. Toublan, and J. Verbaarschot, Kaon condensation and Goldstone's theorem, Phys. Lett. B 522, 67 (2001).

[19] A. Nicolis and F. Piazza, Implications of Relativity on Nonrelativistic Goldstone Theorems: Gapped Excitations at Finite Charge Density, Phys. Rev. Lett. 110, 011602 (2013); 110, 039901(A) (2013).

[20] H. Watanabe, T. s. Brauner, and H. Murayama, Massive Nambu-Goldstone Bosons, Phys. Rev. Lett. 111, 021601 (2013).

[21] A. Nicolis, R. Penco, F. Piazza, and R. A. Rosen, More on gapped Goldstones at finite density: More gapped Goldstones, J. High Energy Phys. 11 (2013) 055.

[22] S. Endlich, A. Nicolis, and R. Penco, Spontaneously broken mass, J. High Energy Phys. 01 (2015) 146.

[23] I. Arav, I. Hason, and Y. Oz, Spontaneous Breaking of nonrelativistic scale symmetry, J. High Energy Phys. 10 (2017) 063.

[24] D. J. Amit and E. Rabinovici, Breaking of scale invariance in $\phi^{6}$ theory: Tricriticality and critical end points, Nucl. Phys. B257, 371 (1985).

[25] E. Rabinovici, B. Saering, and W. A. Bardeen, Critical surfaces and flat directions in a finite theory, Phys. Rev. D 36, 562 (1987).

[26] Z. Komargodski and A. Schwimmer, On renormalization group flows in four dimensions, J. High Energy Phys. 12 (2011) 099.

[27] N. Chai, S. Chaudhuri, C. Choi, Z. Komargodski, E. Rabinovici, and M. Smolkin, Thermal order in conformal theories, Phys. Rev. D 102, 065014 (2020).

[28] I. M. Hayes, R. D. McDonald, N. P. Breznay, T. Helm, P. J. W. Moll, M. Wartenbe, A. Shekhter, and J. G. Analytis, Scaling between magnetic field and temperature in the high-temperature superconductor bafe2(as1-xpx)2, Nat. Phys. 12, 916 (2016).

[29] S. Weinberg, The Quantum Theory of Fields. Vol. 2: Modern Applications (Cambridge University Press, Cambridge, England, 2013).

[30] S. Weinberg, Approximate Symmetries and PseudoGoldstone Bosons, Phys. Rev. Lett. 29, 1698 (1972).

[31] B. Bajc and A. R. Lugo, On the matching method and the Goldstone theorem in holography, J. High Energy Phys. 07 (2013) 056.

[32] C. Hoyos, U. Kol, J. Sonnenschein, and S. Yankielowicz, The holographic dilaton, J. High Energy Phys. 10 (2013) 181.

[33] R. Argurio, D. Musso, and D. Redigolo, Anatomy of new SUSY breaking holographic RG flows, J. High Energy Phys. 03 (2015) 086.

[34] I. Amado, D. Arean, A. Jimenez-Alba, K. Landsteiner, L. Melgar, and I. S. Landea, Holographic Type II Goldstone bosons, J. High Energy Phys. 07 (2013) 108.

[35] R. Argurio, A. Marzolla, A. Mezzalira, and D. Naegels, Note on holographic nonrelativistic Goldstone bosons, Phys. Rev. D 92, 066009 (2015).

[36] G. Cuomo, A. Esposito, E. Gendy, A. Khmelnitsky, A. Monin, and R. Rattazzi, Gapped Goldstones at the cut-off scale: A non-relativistic EFT, arXiv:2005.12924.

[37] N. E. Hussey, Phenomenology of the normal state in-plane transport properties of high-tccuprates, J. Phys. Condens. Matter 20, 123201 (2008).

[38] Z. Leong, K. Limtragool, C. Setty, and P. W. Phillips, Scale invariance as the cause of the superconducting dome in the cuprates, Phys. Rev. B 98, 184501 (2018).

[39] S. Sachdev and B. Keimer, Quantum criticality, Phys. Today 64, No. 2, 29 (2011).

[40] T. Senthil, Deconfined quantum critical points, Science 303, 1490 (2004).

[41] S. Sachdev, Where is the quantum critical point in the cuprate superconductors? Phys. Status Solidi B 247, 537 (2010).

[42] H. Georgi, Unparticle Physics, Phys. Rev. Lett. 98, 221601 (2007).

[43] D. Belitz, T. Kirkpatrick, and T. Vojta, How generic scale invariance influences quantum and classical phase transitions, Rev. Mod. Phys. 77, 579 (2005).

[44] Y. Nishida and D. T. Son, Unitary Fermi gas, epsilon expansion, and nonrelativistic conformal field theories, Lect. Notes Phys. 836, 233 (2012).

[45] K. Ohashi, T. Fujimori, and M. Nitta, Conformal symmetry of trapped Bose-Einstein condensates and massive NambuGoldstone modes, Phys. Rev. A 96, 051601 (2017).

[46] H. Watanabe and A. Vishwanath, Criterion for stability of Goldstone modes and Fermi liquid behavior in a metal with broken symmetry, Proc. Natl. Acad. Sci. U.S.A. 111, 16314 (2014).

[47] D. Musso, Simplest phonons and pseudo-phonons in field theory, Eur. Phys. J. C 79, 986 (2019).

[48] D. Musso and D. Naegels, Independent Goldstone modes for translations and shift symmetry from a real modulated scalar, Phys. Rev. D 101, 045016 (2020).

[49] R. Argurio, C. Hoyos, D. Musso, and D. Naegels (to be published). 
[50] L. V. Delacrétaz, B. Goutéraux, S. A. Hartnoll, and A. Karlsson, Bad metals from fluctuating density waves, SciPost Phys. 3, 025 (2017).

[51] A. Amoretti, D. Areán, R. Argurio, D. Musso, and L. A. Pando Zayas, A holographic perspective on phonons and pseudo-phonons, J. High Energy Phys. 05 (2017) 051.

[52] A. Esposito, S. Garcia-Saenz, A. Nicolis, and R. Penco, Conformal solids and holography, J. High Energy Phys. 12 (2017) 113.

[53] M. Baggioli, V. C. Castillo, and O. Pujolas, Scale invariant solids, Phys. Rev. D 101, 086005 (2020).

[54] L. Alberte, M. Ammon, A. Jiménez-Alba, M. Baggioli, and O. Pujolàs, Holographic Phonons, Phys. Rev. Lett. 120, 171602 (2018).

[55] T. Andrade and B. Withers, A simple holographic model of momentum relaxation, J. High Energy Phys. 05 (2014) 101.

[56] A. Donos and J.P. Gauntlett, Holographic Q-lattices, J. High Energy Phys. 04 (2014) 040.
[57] A. Amoretti, D. Areán, B. Goutáraux, and D. Musso, Effective holographic theory of charge density waves, Phys. Rev. D 97, 086017 (2018).

[58] A. Amoretti, D. Areán, B. Goutéraux, and D. Musso, DC Resistivity of Quantum Critical, Charge Density Wave States from Gauge-Gravity Duality, Phys. Rev. Lett. 120, 171603 (2018).

[59] A. Donos, J.P. Gauntlett, T. Griffin, and V. Ziogas, Incoherent transport for phases that spontaneously break translations, J. High Energy Phys. 04 (2018) 053.

[60] M. Baggioli, V. V. Brazhkin, K. Trachenko, and M. Vasin, Gapped momentum states, Phys. Rep. 865, 1 (2020).

[61] A. Amoretti, D. Areán, B. Goutéraux, and D. Musso, Diffusion and universal relaxation of holographic phonons, J. High Energy Phys. 10 (2019) 068.

[62] A. Amoretti, D. Areán, B. Goutéraux, and D. Musso, Gapless and gapped holographic phonons, J. High Energy Phys. 01 (2020) 058. 